\title{
Numerical heating of electrons in particle-in-cell simulations of fully magnetized plasmas
}

\author{
M. Horký* \\ Institute of Atmospheric Physics, Czech Academy of Sciences, Boční II 1401, 14100 Prague, Czech Republic \\ and Astronomical Institute, Czech Academy of Sciences, Boční II 1401, 14100 Prague, Czech Republic \\ W. J. Miloch \\ Department of Physics, University of Oslo, Box 1048 Blindern, N-0316 Oslo, Norway \\ V. A. Delong \\ Faculty of Electrical Engineering, Czech Technical University in Prague, Technická 2, 16627 Prague, Czech Republic
}

(Received 3 August 2016; revised manuscript received 7 January 2017; published 12 April 2017)

\begin{abstract}
The role of spatial resolution of the electron gyroradius in electrostatic particle-in-cell (PIC) simulations is studied. It is demonstrated that resolving the gyroradius is crucial for simulations of strongly magnetized plasmas and that nonresolving it results in substantial anisotropic heating of electrons. The numerical heating can, in some cases, be suppressed by the higher-order weighting to the grid, but it cannot be avoided. Possible mechanisms behind this numerical heating are discussed. The study is carried out with a fully three dimensional electrostatic PIC code with an external magnetic and electric fields.
\end{abstract}

DOI: 10.1103/PhysRevE.95.043302

\section{INTRODUCTION}

Numerical simulation is a common approach for studying dynamical plasma phenomena. The plasma models usually depend on the spatiotemporal scales of interest and are reflected in numerical approaches. Magnetohydrodynamic and fluid simulations consider plasma as conducting fluids. They are concerned with slow and large-scale phenomena, thus assuming that the simulated plasma velocity is the average over the velocity phase space [1]. In another limit, the Vlasov codes are concerned with solving the Vlasov equation to study the evolution of the plasma velocity distribution function [2]. For studying plasma at a kinetic level, at small and intermediate scales, the most common numerical approach is the particle-in-cell (PIC) numerical method $[3,4]$.

In the PIC method a large number of numerical particles representing electrons and ions are simulated and their trajectories are followed in self-consistent force fields. To reduce the numerical complexity and make the large-scale simulations feasible, the simulated particles interact with each other via a computational grid that is used to calculate the force field $[3,4]$. The use of the grid reduces the complexity of numerical operations from $\mathcal{O}\left(N^{2}\right)$, where $N$ is the number of simulated particles and $N^{2}$ relates to evaluation of forces between particle pairs, down to $\mathcal{O}\left(N \log N_{\mathrm{g}}\right)$, where $N_{\mathrm{g}}$ is the number of grid points, $N_{\mathrm{g}} \ll N$ and $N$ is now related to the particle-grid operations (weighting, force projection). Note that the equations of motion for the simulated particles are the characteristics of the Vlasov equation, and the PIC method can be related to solving this equation with the method of characteristics. While the PIC method gives, in general, much more noisy results than the Vlasov simulations due to the finite number of simulated particles, it offers a powerful tool due to its flexibility and computational efficiency [4].

*mh@ufa.cas.cz
In the standard, explicit PIC method, the trajectories of simulated particles are usually advanced with the leap-frog or Boris algorithms [3,4]. The leap-frog method is characterized by a staggered time-mesh for velocities $\mathbf{v}$ and positions $\mathbf{x}$ :

$$
\begin{aligned}
\mathbf{x}_{\mathbf{i}}(\mathbf{t}+\Delta \mathbf{t}) & =\mathbf{x}_{i}(t)+\mathbf{v}_{i}(t+\Delta t / 2) \Delta t, \\
\mathbf{v}_{i}(t+\Delta t / 2) & =\mathbf{v}_{i}(t-\Delta t / 2)+\mathbf{F}_{i}(t) \Delta t / m_{i},
\end{aligned}
$$

where $i$ refers to a plasma particle, $\mathbf{F}_{i}=q_{i} \mathbf{E}$ is the electric force projected on the $i$ th particle from the nearest grid points, and $\Delta t$ is the computational time step. The Boris algorithm $[5,6]$ accounts for the velocity rotation due to the Lorentz force $\mathbf{F}_{\mathrm{L}}=q(\mathbf{E}+\mathbf{v} \times \mathbf{B})$. It rotates velocity $\mathbf{v}_{-}$in the plane perpendicular to the magnetic field $\mathbf{B}=m \hat{\Omega} /|q|$, where $\hat{\Omega}$ is the gyrofrequency, to a new velocity $\mathbf{v}_{+}$:

$$
\begin{aligned}
\mathbf{w} & =\mathbf{v}_{-}+\mathbf{v}_{-} \times \boldsymbol{\xi}, \\
\mathbf{v}_{+} & =\mathbf{v}_{-}+\mathbf{w} \times \boldsymbol{\eta},
\end{aligned}
$$

where $\xi=\hat{\Omega} \tan (\Omega \Delta t / 2)$, and $\boldsymbol{\eta}=2 \xi /\left(1+\xi^{2}\right)$. To combine the two algorithms, one needs to first half-accelerate the particle due to the electric force (i.e., advance its velocity for a half-time step $\Delta t / 2$ ), rotate the velocity due to the magnetic field according to Eq. (2), and again half-accelerate the particle due the electric force using Eq. (1). Thus the mean particle velocity is used for the Lorentz force calculation.

The force field is calculated with a difference scheme on the spatial grid from the particle data weighted to the grid, and then projected to the particles. This particle-grid interaction introduces the weighting shape factors $S$, which depend on the details of weighting routines, usually employing B-splines. The commonly used weighting is the first-order linear interpolation $S_{1}$ (e.g., in Refs. [7-10]), which distributes the charge density between the nearest gridpoints [two points in the one-dimensioanl (1D) case]. The second-order (quadratic) spline $S_{2}$ which assigns the charge density between more gridpoints (three nearest points in the 1D case), and the nearest grid point (NGP) scheme, $S_{0}$ are used less frequently $[11,12]$. The 
second-order weighting smoothes charge density distribution and can supress numerical instabilities to the certain level [13], however, it is computationally more expensive. On the other hand, the NGP is very fast but it is characterized by an increased noise level. Therefore, as a compromise, the $S_{1}$ is usually chosen due to its effective shape factor [14].

The use of a spatiotemporal grid implies restrictions on the time step and grid spacing used in the simulations [15]. The numerical accuracy of the explicit temporal integration (1) requires that the time step $\Delta t<\omega^{-1}$, where $\omega$ is the largest characteristic frequency in the system (usually the plasma frequency or electron gyrofrequency) $[4,16]$. The leap-frog (1) is numerically unstable for $\omega \Delta t \geqslant 2$. For the range $1<$ $\omega \Delta t<2$ the scheme is stable, however, the numerical error is considerable. To conserve the energy in the PIC simulation, it is typically advised to use $\omega \Delta t \leqslant 0.1$ [15].

Using a spatial grid leads to condition $\Delta x<\gamma \lambda_{\mathrm{D}}$, where $\gamma$ is the numerical factor that depends on the order of weighting of the fields to the grid points (for the first-order weighting $\gamma=\pi$ ) and $\lambda_{\mathrm{D}}$ is the Debye length which is related to the electron temperature as $\lambda_{\mathrm{D}} \sim \sqrt{T_{\mathrm{e}}}$. If this condition is not satisfied, aliasing with the grid will lead to heating of plasma. The heating will stop when the condition is fulfilled, i.e., when $\lambda_{\mathrm{D}} \approx \gamma^{-1} \Delta x$. Note that this condition can be generalized as $v_{\mathrm{th}} \Delta t \ll \Delta x$, thus the particles should not move more than one grid cell within $\Delta t$, which implies that the particle information should be accurately represented on the grid. In electromagnetic codes the previous conditions lead to the Courant-Friedrichs-Lewy (CFL) criterion, stating that the propagation of electromagnetic waves must fulfill $c \Delta t<\Delta x$.

In the studies of spatial grid effects, most of the efforts have been put on the resolution of $\lambda_{D}$ [13]. However, in strongly magnetized plasmas one can have electron gyroradius $r_{\mathrm{L}}<\lambda_{\mathrm{D}}[17,18]$. It is generally accepted that not resolving the electron gyroradius on the spatial grid (when $r_{\mathrm{L}}<\Delta x$ ) does not have consequences on the numerical stability due to the homogenous distribution of the particles and that the accurate resolving the gyration by temporal spacing is sufficient. Birdsall and Langdon [4] noted that smoothing the distribution function by not resolving the Larmor radius, for example, when $r_{\mathrm{L}} \leqslant \Delta x$, will have a stabilizing effect when considering the numerical instability of the magnetized plasma due to a finite time step $\Delta t$ [16]. Only recently has it been noted that unresolved $r_{\mathrm{L}}$ might lead to plasma heating, and that this parameter regime should be avoided $[17,19]$. Thus it remains an open question whether the electron gyroradius needs to be resolved in PIC simulations and what are the consequences of the gyroradius resolution for the system stability.

In this paper we study the effects of $r_{\mathrm{L}}$ resolution in a PIC simulation of strongly magnetized plasma. We demonstrate that for unresolved Larmor radius the electrons are being heated in the direction perpendicular to the magnetic field. These effects can be only partially diminished, but cannot be avoided by using higher-order weighting functions.

\section{SIMULATION DESCRIPTION}

For our simulations we use a three-dimensional (3D) momentum-conserving electrostatic code called DIP3D, which was developed at the University of Oslo and was used many times before (e.g., Refs. [20-22]). Particles are advanced with the leap-frog method [Eq. (1)] combined with the Boris algorithm [Eq. (2)] and electric potential is calculated using the multigrid method. We use the Cartesian coordinate system, and can choose between the first- and second-order weightings of particles and fields [4]. DIP3D also allows to simulate collisions between charged particles and neutrals using the "null collision method" [23,24], external electric fields, as well as charging of dust grains and other objects in plasma [22], however, these features are not used in this study.

We set the external magnetic field $\mathbf{B}$ in the $\hat{\mathbf{x}}$ direction and use a cubic simulation box with periodic boundaries. To account for different spacing, we modify the length of each side and the number of grid cells. We use the Debye length and plasma density as input parameters, from which we derive both the plasma frequency and the temperature. Thus to vary the initial temperature and thermal gyroradius we can change either the Debye length or density. In all simulations we resolve the electron Debye length in the spatial domain and all characteristic frequencies in the temporal domain. From diagnostics we focus on the velocity phase space and electric potential distributions. At given time instances we can reconstruct the electron distribution function, from which we are able to estimate the electron temperature using the fact that the second moment of the Maxwellian distribution corresponds to the temperature by the relation

$$
\sigma_{\mathrm{v}}^{2}=v_{\mathrm{th}}^{2}=\frac{k_{\mathrm{B}} T}{m} .
$$

The code can save the electric potential of a grid or subgrid during the simulation which allows for studying the temporal evolution of potential fluctuations as well as frequency wave number spectra in all three directions.

In this study we typically simulate $N \propto 10^{6}$ particles per plasma species. Parallelization is done using particle redistribution over different nodes, and the message-passinginterface (MPI) is used for the parallel computation. In PIC simulations one should be aware of having a sufficient average number of particles per cell $\left(N_{\mathrm{c}}\right)$ because the initial level of potential fluctuation scales with $1 / \sqrt{N_{\mathrm{c}}}$. We verified our results by varying the number of particles per cell [25], and found that the current setup is appropriate and that further increase of $N_{\mathrm{c}}$ does not change the evolution of the system.

We use different simulation setups. In each of them we have one simulation with the first-order weighting $S_{1}$ and one with the second-order weighting $S_{2}$. These simulations are labeled A1, A2, B1, B2, C1, C2, D1, and D2, where the capital letters stand for the setup and the numbers correspond to the weighting order. The simulation parameters are summarized in Table I. In these four sets (A, B, C, and D) we use a different resolution of gyroradius on the grid. Simulations A have highly unresolved gyroradius. Simulations B also have an unresolved gyroradius, but the length of the simulation box is much smaller, while the number of grid points is chosen to have the same spacing as in simulations A. This is to study whether the numerical heating does depend on the size of the box. Simulations $\mathrm{C}$ have a well-resolved gyroradius and simultions $\mathrm{D}$ have grid spacing similar to the gyroradius. Thus our simulations cover three main cases: (i) unresolved gyroradus, (ii) well-resolved gyroradius, and (iii) gyroradius similar to 
TABLE I. Parameters used in simulations of magnetized plasmas. Numbers 1 or 2 in each setup stands for order of weighting function $S_{1}$ and $S_{2}$, respectively. In all simulations we used the external magnetic field $\mathbf{B}_{0}=0.005 \hat{x}$ T, electron Debye length $\lambda_{\mathrm{D}}=9.76 \times 10^{-3} \mathrm{~m}$, temperature ratio $T_{\mathrm{e}} / T_{\mathrm{i}}=4$, and mass ratio $m_{\mathrm{i}} / m_{\mathrm{e}}=500$.

\begin{tabular}{lcccc}
\hline \hline Param. $\backslash$ Sim. & A1 \& A2 & B1 \& B2 & C1 \& C2 & D1 \& D2 \\
\hline$L_{\mathrm{x}}, L_{\mathrm{y}}, L_{\mathrm{z}}$ & $0.5 \mathrm{~m}$ & $0.125 \mathrm{~m}$ & $0.125 \mathrm{~m}$ & 128 \\
$n_{\mathrm{gx}}, n_{\mathrm{gy}}, n_{\mathrm{gz}}$ & 64 & 16 & $9.77 \times 10^{-4} \mathrm{~m}$ & 128 \\
$\Delta x, \Delta y, \Delta z$ & $7.81 \times 10^{-3} \mathrm{~m}$ & $7.81 \times 10^{-3} \mathrm{~m}$ & $3.9 \times 10^{-10} \mathrm{~s}$ & $3.91 \times 10^{-3} \mathrm{~m}$ \\
$\Delta t$ & $5 \times 10^{-10} \mathrm{~s}$ & $5 \times 10^{-10} \mathrm{~s}$ & $5 \times 10^{12} \mathrm{~m}^{-3}$ & $2.5 \times 10^{-10} \mathrm{~s}$ \\
$n_{\mathrm{i}}, n_{\mathrm{e}}$ & $5 \times 10^{12} \mathrm{~m}^{-3}$ & $5 \times 10^{12} \mathrm{~m}^{-3}$ & $2 \times 10^{7}$ & $4.3 \times 10^{13} \mathrm{~m}^{-3}$ \\
$N_{\mathrm{i}}, N_{\mathrm{e}}$ & $5 \times 10^{6}$ & 78125 & 18.3 & $2 \times 10^{7}$ \\
$2 \pi / \Omega_{\mathrm{ce}} \Delta t$ & 14.3 & 14.3 & 127.9 & 28.6 \\
$2 \pi / \omega_{\mathrm{pe}} \Delta t$ & 99.7 & 99.7 & 9.99 & 68.1 \\
$\lambda_{\mathrm{D}} / \Delta x$ & 1.249 & 1.249 & 1.43 & 1.05 \\
$v_{\mathrm{th}} / \Omega_{\mathrm{ce}} \Delta x$ & 0.18 & 0.18 & $8.62 \mathrm{eV}$ & $74.12 \mathrm{eV}$ \\
$T_{\mathrm{e}}$ & $8.62 \mathrm{eV}$ & $8.62 \mathrm{eV}$ & \\
\hline \hline
\end{tabular}

spacing. By comparing results from these three cases we can obtain the qualitative description of numerical heating due to the resolution of the gyroradius. Note that in all simulations the time step is sufficiently small to resolve the electron gyromotion in the time domain. Thus the Boris algorithm that is used to advance particle trajectories and velocities is stable.

As an additional case we extend our analysis by including also external electric field. The electric field is in a direction perpendicular to the magnetic field and gives rise to the $\mathbf{E} \times \mathbf{B}$ drift of plasma. The strength of the added electric field is $550 \mathrm{Vm}^{-1}$ and drift velocity is $v_{\mathrm{D}}=11000 \mathrm{~ms}^{-1}$. These values are chosen to obtain supersonic drift velocity and still to have stable plasma due to the absence of relative drift speed between species (ions are also fully magnetized). Plasma parameters of this simulation correspond to the simulation D while the grid spacing is twice as large, thus the gyroradius is unresolved. This extra case is considered to verify whether the flowing magnetized plasma will have similar characteristics as the stationary magnetized plasma.

\section{SIMULATION RESULTS}

For all simulation runs shown in Table I we fulfilled all standard stability criteria such as the temporal resolution of the highest system frequencies and spatial resolution of the electron Debye length. Thus the only varying parameter is the resolution of the electron gyroradius. In the analysis of the numerical results we focus on the temporal evolution of the electron temperature, evolution of potential fluctuations, and wave spectra.

Temporal evolutions of the electron temperature for each case are shown in Fig. 1. Since the gyroradius is calculated from the perpendicular thermal velocity, the $r_{\mathrm{L}} / \Delta x$ ratio can be attributed to the perpendicular temperature, therefore we also show a scale with this ratio. The left panels show temperatures in each direction for the simulations with the first-order weighting $S_{1}$ and the right panels show results for cases with the second-order weighting $S_{2}$. In simulations A1 and A2 we have highly unresolved electron gyroradius $r_{\mathrm{L}} / \Delta x \approx 0.18$ and well-resolved gyrofrequency $2 \pi / \Omega_{\mathrm{ce}} \Delta t \approx 14.3$. We observe heating of electrons in the perpendicular directions for case A1. The heating starts approximately after 80 plasma periods.
For the case with the second-order weighting A2, we do not observe any significant heating and the temperatures only fluctuate around their initial values.

The temperature increase may be reflected in the temporal evolution of potential fluctuations. The potential fluctuations for both runs are shown in Fig. 2(a). For the case with the firstorder weighting we observe an increase in root mean square (RMS) of potential fluctuations: their level doubles during the simulation. However, for the case with the second-order weighting we see a slight decrease in RMS values, indicating smoothing of the potential. The oscillations in RMS values visible at the beginning of the simulation are related to the ion plasma oscillations. To further study the potential fluctuations we present in Fig. 3 snapshots of the potential density in the plane perpendicular to the magnetic field at different time instances corresponding to the initial state, growing phase, and the later stage. A striking feature is the growth of potential filaments aligned with the magnetic field lines, which give rise to strong density gradients in the perpendicular directions as well as growth in the amplitudes.

In view of our results, it may be instructive to analyze also the wave spectra. We present spectra for the perpendicular direction obtained from the whole simulation run in Fig. 4 (upper panels for A1). For clarity we show spectra of potential fluctuations around frequencies where there is the largest activity. In the left-upper panel we see activity around the upper hybrid frequency with several harmonics, which is typical for the perpendicular direction in magnetized plasmas. In the rightupper panel, there are oscillations on the ion plasma frequency that dominate. We note that the signature of the ion plasma oscillations becomes less visible at the later stage of the simulation, which agrees with the results for the potential evolution.

In simulations B1 and B2 we use the same plasma parameters, and the same grid spacing as in setups A1 and A2. However, the size of the simulation box is different to study if the box size can affect electron heating. The temporal evolution of electron temperature is shown in Fig. 1 (B1,B2) and it is clear that the box size has a negligible effect on the evolution of the electron temperature: the heating in perpendicular directions has a similar rate as the heating in simulation A1. Similarly to simulations A2, in simulations B2 we do not observe any heating for the case with the 

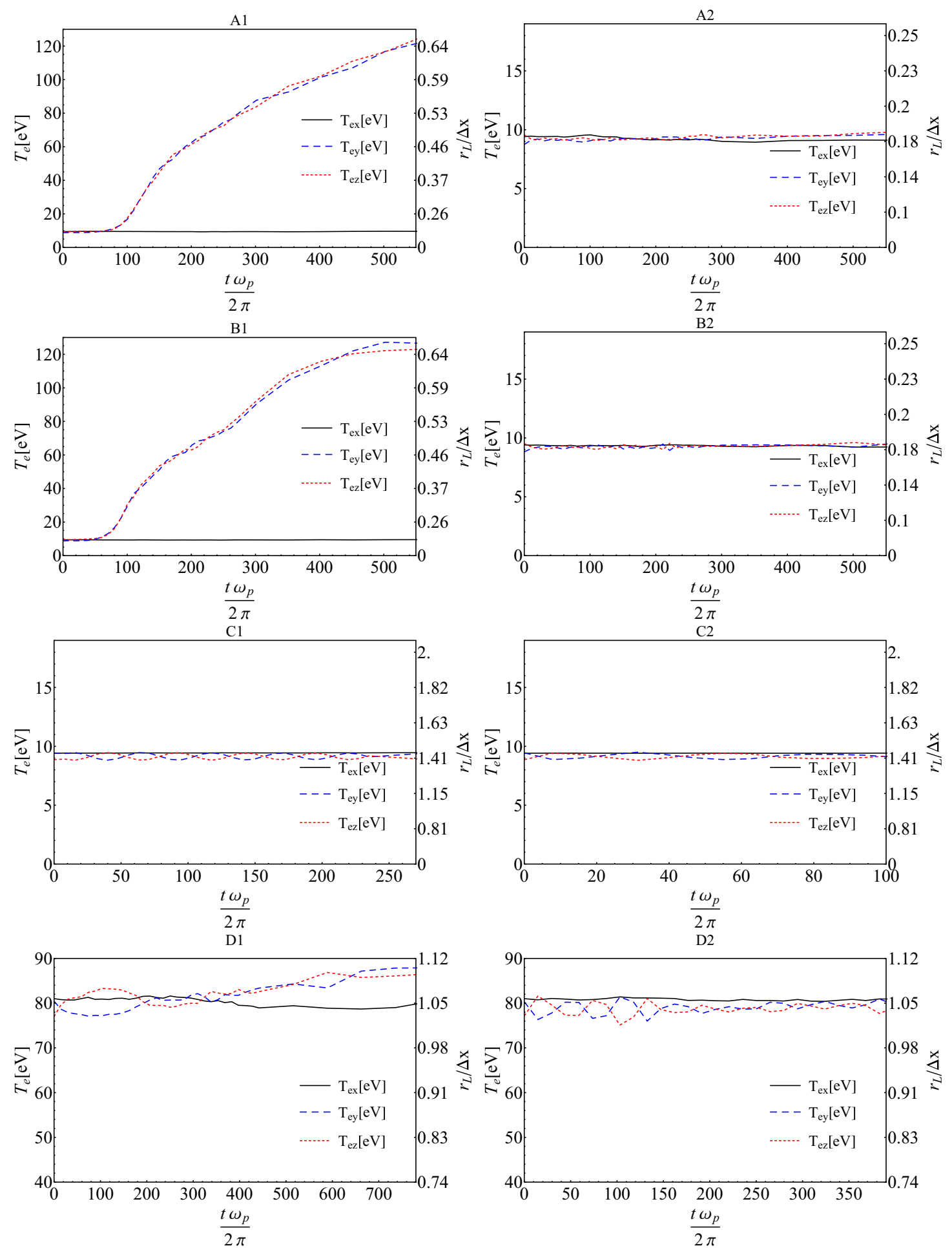

FIG. 1. The electron temperature evolution for all simulation runs. The first-order weighting is given in the left column, while the second-order weighting is in the right column. Magnetic field is oriented in the $x$ direction. The scale on right side corresponds to the $r_{\mathrm{L}} / \Delta x$ ratio.

second-order weighting. A different situation is in the case of potential fluctuations. It is obvious from Fig. 2(b) that the initial fluctuation is lower in cases B than in simulations A1 and A2. It is because the number of simulated particles is the same as in simulations A, but the volume of one elementary cell $(\Delta x \times \Delta y \times \Delta z)$ is much smaller than in the previous case. For the first-order weighting we observe exponential growth of fluctuations starting after approximately 40 electron plasma periods and lasting until approximately 130 plasma periods when the growth becomes smaller. At the end of the simulation, the fluctuations reach a level that is similar to those in simulations A1. The evolution of potential fluctuations in 

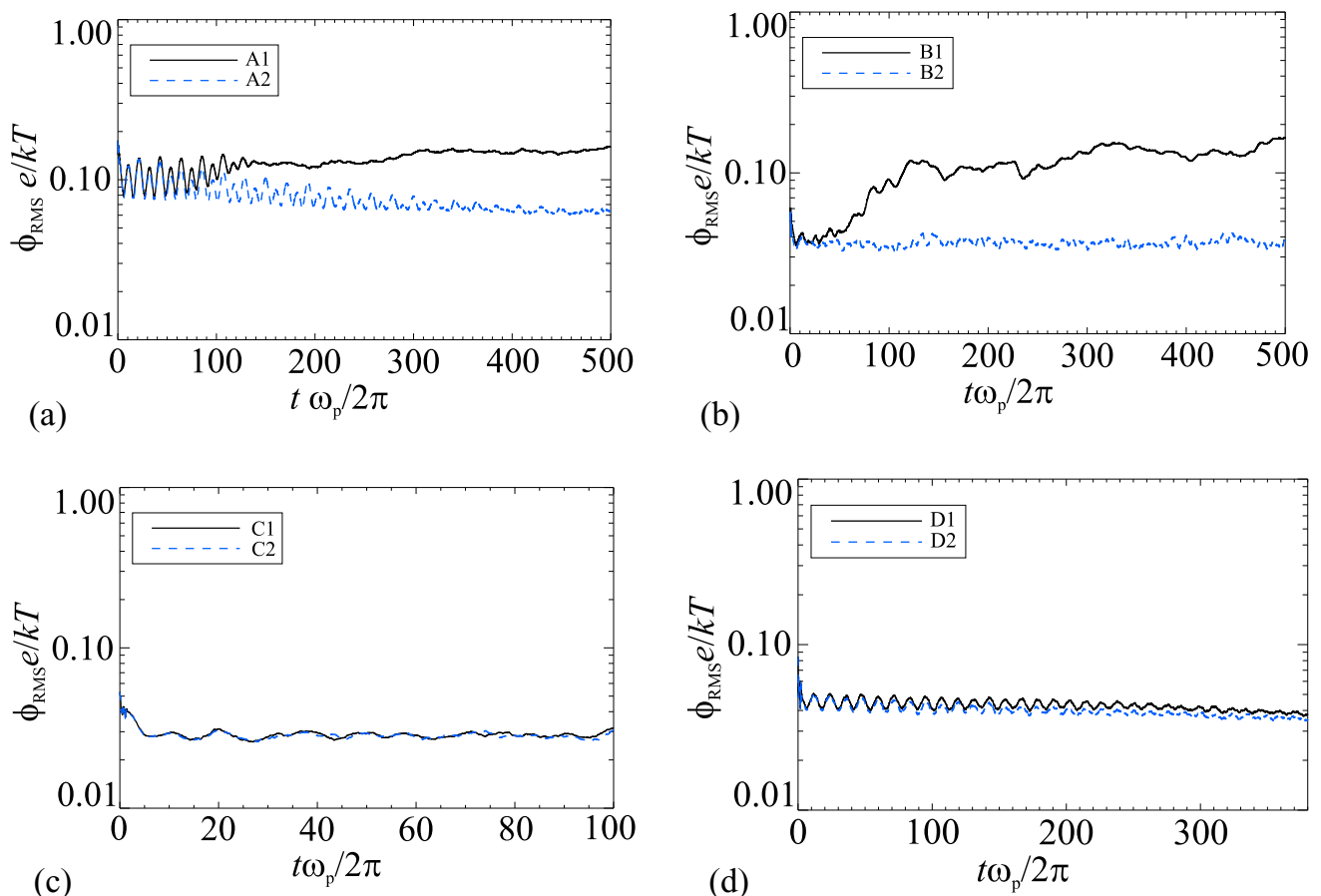

FIG. 2. Temporal evolution of RMS values of normalized potential fluctuations. In each figure the simulation with the first-order weighting is depicted by solid black line and the case with the second-order weighting by dashed blue line. Note that the vertical scale in panel (c) is different than in other panels.

simulations B2 shows damping, similar to simulations A2. We checked also the potential density in the plane perpendicular to the magnetic field, and just like in setup A, the potential created filaments along the magnetic field. Thus we present spectra of waves in potential fluctuations also for simulation B1 in Fig. 4(B1). Note that the normalization factors for spectra A1 and $\mathrm{B} 1$ are different and each spectra are normalized to their own maxima. While we cannot compare depicted amplitudes between the two setups we can still assess the shape of the spectra. Such a comparison shows that there are no significant oscillations on the ion plasma frequency for B1, but we still observe the wave activity around the upper hybrid frequency.

In simulations $\mathrm{C} 1$ and $\mathrm{C} 2$ we have a very good resolution of the electron gyroradius. We do not observe any growth in the electron temperature in Fig. 1 (cases $\mathrm{C} 1$ and $\mathrm{C} 2$ ) nor in the fluctuations of potential in Fig. 2(c). The electron temperature only slightly fluctuates around initial values and the RMS values of the electric potential stay at a low level during the whole simulation. We note that to maintain the scales for potential evolution figures, the RMS values of potential fluctuations are multiplied by 10 in Fig. 2(c). There is no significant difference between the runs with the first-order and the second-order weighting in setup $\mathrm{C}$. In the wave spectra we did not observe any significant wave or oscillation activity.

Simulations D1 and D2 are set to have the same box size as simulations $\mathrm{A} 1$ and $\mathrm{A} 2$ and the resolution of gyroradius slightly above $v_{\mathrm{th}} / \Omega_{\mathrm{ce}} \Delta x=1$, thus they can be assumed as a case with the minimal resolution of gyroradius. We note that these simulations have warmer electrons from the beginning. It is because in the DIP3D code the temperature of particles is calculated from the Debye length and number density which are defined as input parameters. To have a larger thermal gyroradius we need to increase either the Debye length or density or decrease the magnetic field. To keep the same Debye length and magnetic field in all simulation runs, we therefore increased the density up to the order $n_{\mathrm{i}, \mathrm{e}} \sim 10^{13}$, thus we get the required resolution of gyroradius. The temporal evolution of the electron temperature in Fig. 1 (D1,D2) shows fluctuations and little growth of temperature in the perpendicular direction in simulation D1. The results of simulation D2 show stable temperature in the magnetically aligned direction and oscillations in perpendicular directions. The evolution of RMS in the potential distribution in Fig. 2(d) shows only oscillations on the ion plasma frequency for both weightings. The plasma oscillations also dominate the spectra.

Finally, we consider also the additional case with external electric field. In Fig. 5 we show the temporal evolution of electron temperature for both first-order and second-order weightings. A significant difference in this case is that we observe pronounced heating in both cases starting again around after 80 plasma periods. For the first-order weighting, the heating is observed even in the $\hat{\mathbf{x}}$ direction. The second-order weighting does not cancel the heating but only reduces it. However, in both cases, the numerical heating is significant.

In summary, we observe significant changes in the electron temperature in all simulations where we used the first-order weighting and the gyroradius was not well resolved. Thus, except for $\mathrm{C} 1$ and $\mathrm{C} 2$ runs, we observed either growth of the potential fluctuations or potential oscillations. The frequency spectra show that in these cases the dominant waves are around upper-hybrid and electron-cyclotron frequencies and the dominant oscillations are on the ion plasma frequency. For the simulations with the second-order (quadratic) weighting, there was no heating nor growth in the potential fluctuations 

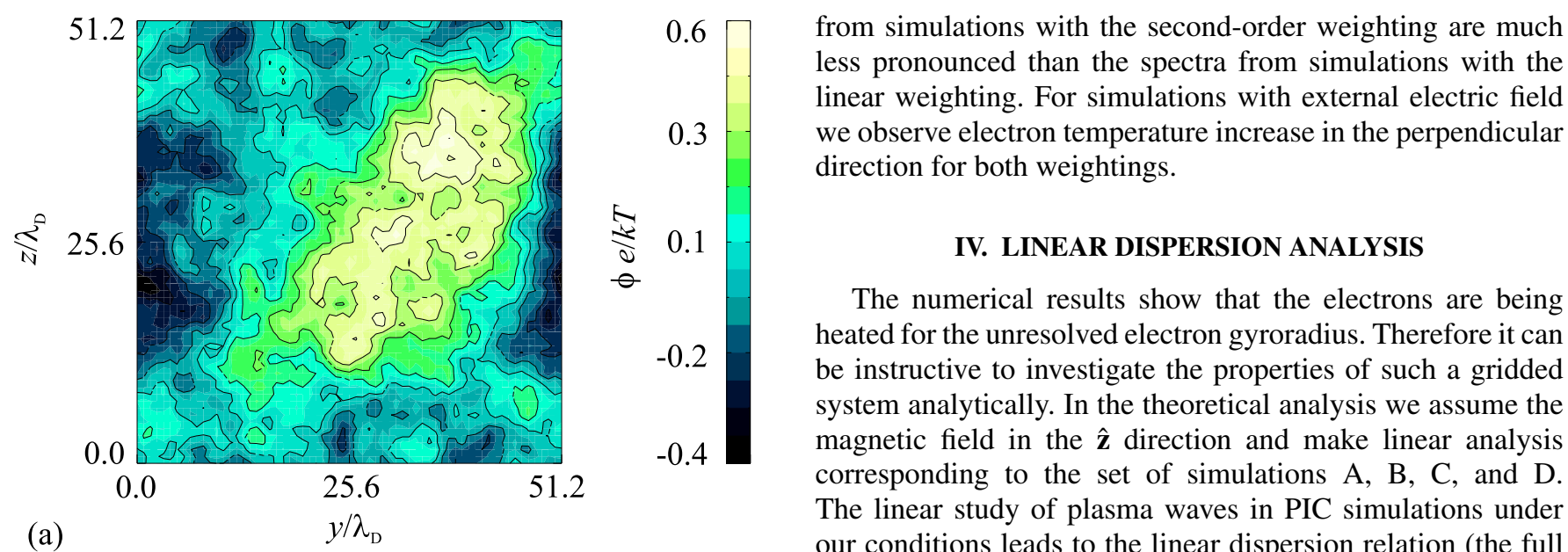

(a)
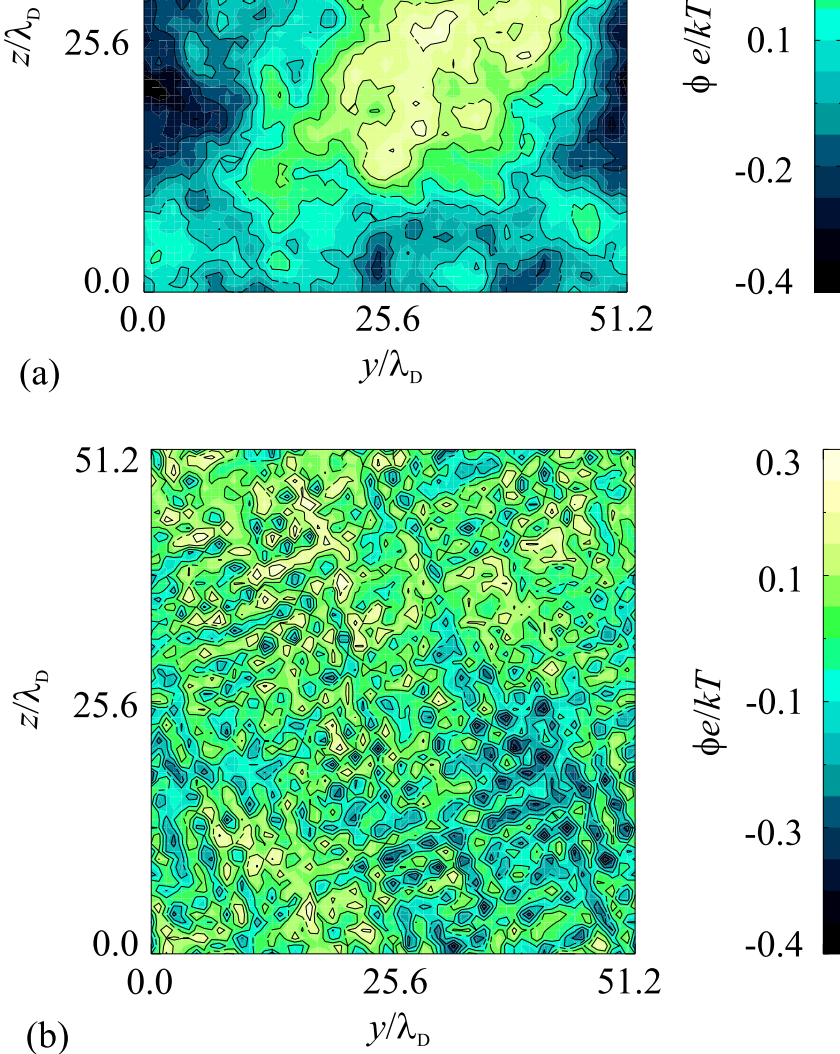

$$
\begin{array}{r}
1+\sum_{\alpha} \sum_{p=-\infty}^{\infty}\left|S\left(\mathbf{k}_{p}\right)\right|^{2} \frac{\omega_{\mathrm{p} \alpha}^{2}}{\mathcal{K}^{2}\left(\mathbf{k}_{p}\right) v_{\mathrm{th} \alpha}^{2}} \\
\left.\times-1 \frac{\omega \exp \left(-\lambda_{\alpha}\right)}{\sqrt{2}\left|\kappa_{\mathrm{z}}\right| v_{\mathrm{th} \alpha}} \sum_{m=-\infty}^{\infty} \mathrm{I}_{m}\left(\lambda_{\alpha}\right) \mathrm{Z}\left(\zeta_{\alpha}^{m}\right)\right]=0,
\end{array}
$$

where

$$
S\left(\mathbf{k}_{p}\right)=\left[\operatorname{dif}\left(\frac{k_{p x} \Delta x}{2}\right) \operatorname{dif}\left(\frac{k_{p y} \Delta y}{2}\right) \operatorname{dif}\left(\frac{k_{p z} \Delta z}{2}\right)\right]^{m+1}
$$

is the shape factor given by the order of weighting. The dif function is the diffraction function defined by the authors of Ref. [4] as

$$
\operatorname{dif} \theta=\frac{\sin \theta}{\theta} .
$$
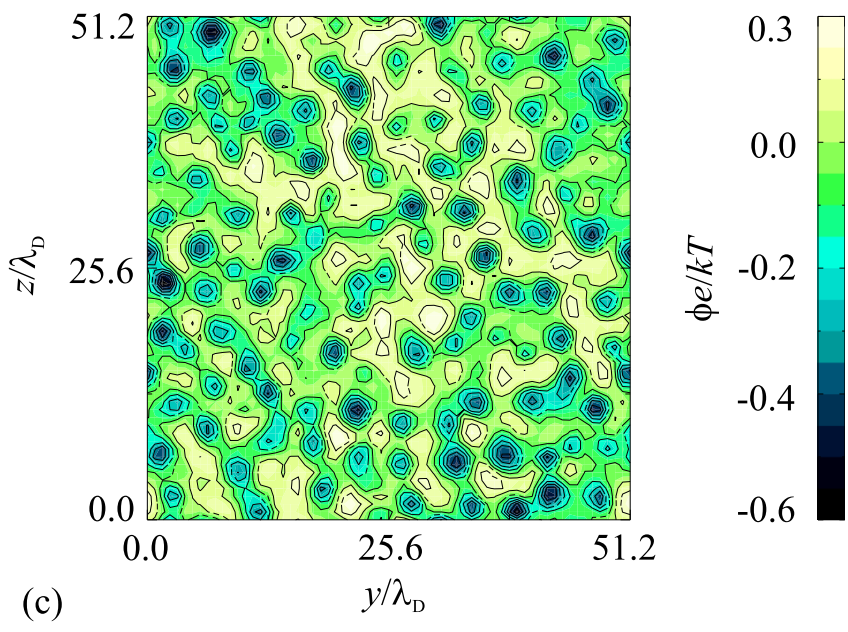

FIG. 3. Cuts in the $y-z$ plane for the potential density showing time evolution of electric potential in the plane perpendicular to the magnetic field in the case A1. Panel (a) corresponds to the initial state of potential, panel (b) shows the cut for the time $t=90 / \omega_{\mathrm{p}}$, and panel (c) shows potential for the time $t=270 / \omega_{\mathrm{p}}$. The evolution of filamentary structures along magnetic field is clearly visible. Both axes are normalized to the Debye length $\lambda_{D}$.

for plasma with no external electric field. We observe only the ion plasma oscillations in simulation D2. The spectra

The definition of variables $\boldsymbol{\kappa}$ and $\mathcal{K}^{2}$ is in Eqs. (A2) and (A3) (according to the authors of Ref. [4]) and their meaning is the effect of the grid on the quantities calculated on the grid (forces, potential, etc.).

The dispersion relation in Eq. (4) provides a solution for the arbitrary direction of the wave vector. Since we observe heating mainly in the direction perpendicular to the magnetic field, we can simplify the dispersion relation to the form which contains only the perpendicular wave vector.

To make this simplification we can use asymptotic expansion of the plasma dispersion function, set the $k_{z}=0$ and get the dispersion relation

$$
\begin{aligned}
1 & +\sum_{\alpha} \sum_{p=-\infty}^{\infty}\left|S\left(\mathbf{k}_{p}\right)\right|^{2} \frac{\omega_{\mathrm{p} \alpha}^{2}}{\mathcal{K}^{2}\left(\mathbf{k}_{p}\right) v_{\mathrm{th} \alpha}^{2}} \\
& \times\left[1-\omega \exp \left(-\lambda_{\alpha}\right) \sum_{m=-\infty}^{\infty} \frac{\mathrm{I}_{m}\left(\lambda_{\alpha}\right)}{\omega+m \Omega_{\alpha}}\right]=0 .
\end{aligned}
$$

As the first approximation we solved this equation in its dimensionless form numerically for $p=0$ using different resolutions of Larmor radius and for the different orders of the shape function. In all cases the obtained solution had no imaginary part, thus there was no growth nor damping, and it contained only real parts of frequency corresponding to Bernstein modes. Thus, the linear analysis did not provide 


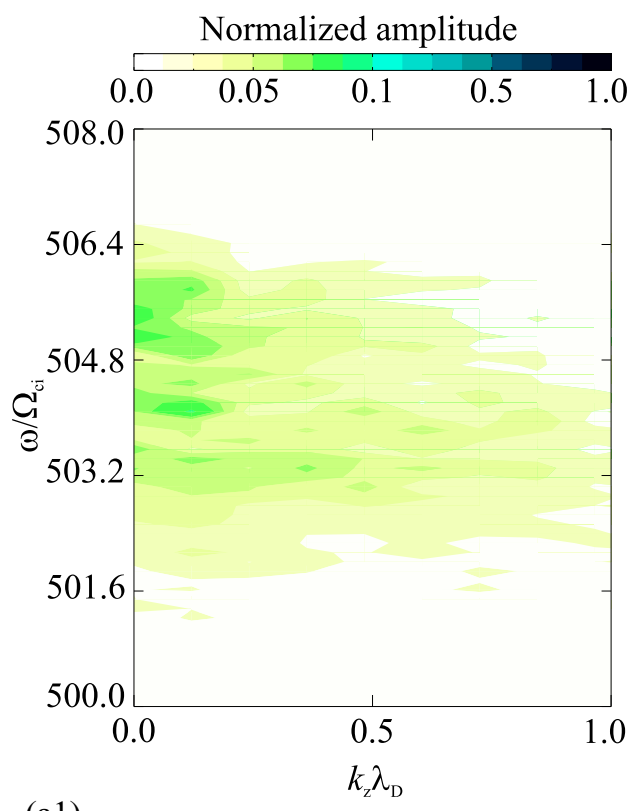

(a1)
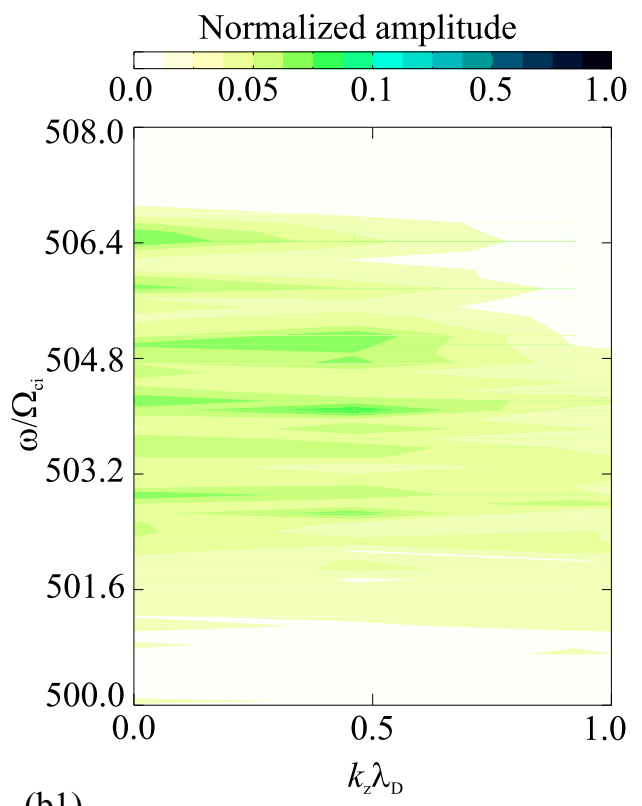

(b1)

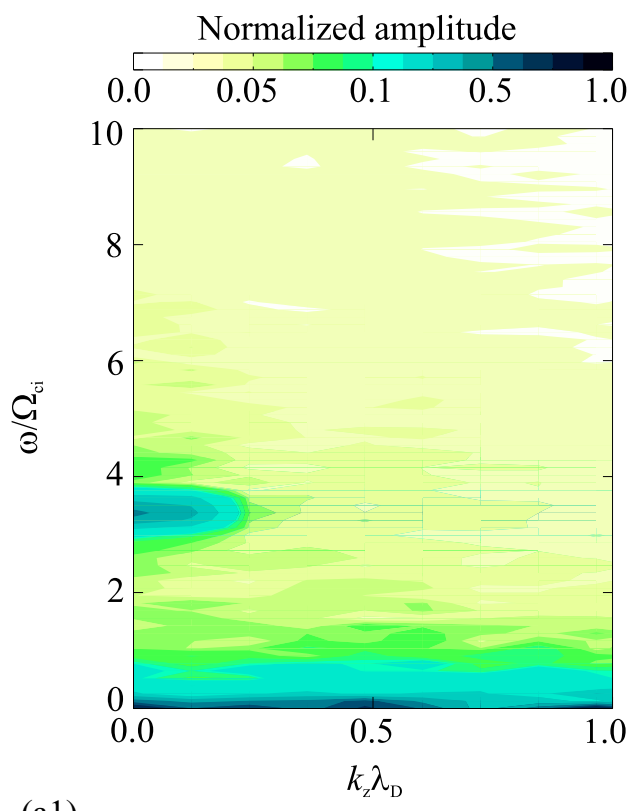

(a1)

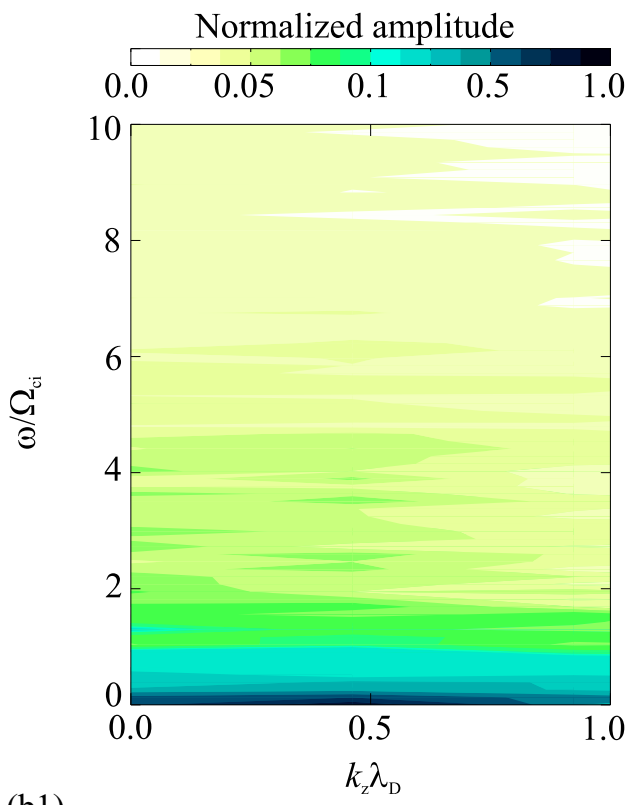

FIG. 4. $\omega-k_{z}$ amplitude spectra of electric potential for simulations resulting in temperature and potential growth. Top panels depict spectra for A1 electron gyro/upper hybrid frequency range (left), and A1 ion gyrofrequency range (right), while bottom panels depict spectra for B1 electron gyro/upper hybrid frequency range (left), and B1 ion gyrofrequency range (right). The frequency axis is normalized to ion gyrofrequency and wave number axis is normalized to the Debye length. We have $\Omega_{\mathrm{e}}=500 \Omega_{\mathrm{i}}$ and $\omega_{\mathrm{uh}} \approx 504.5 \Omega_{\mathrm{i}}$ and thus we can see activity in the upper hybrid frequency range which is typical for perpendicular direction in magnetized plasmas.

an explanation for the electron heating in the perpendicular direction due to the unresolved gyroradius.

\section{DISCUSSION}

The results from this study show that the resolution of the electron gyroradius $r_{\mathrm{L}}$ is a crucial aspect of simulating strongly magnetized plasmas where the electron gyroradius $r_{\mathrm{L}}$ is the smallest characteristic scale. In simulations with the highly unresolved electron gyroradius, we observe significant numerical heating of electrons in the direction perpendicular to the magnetic field. The heating starts after 60-80 electron plasma periods and in the beginning the heating is exponential. After another 70-90 plasma periods, the perpendicular electron temperature growth becomes almost linear. Finally, at later stages the temperature increase goes asymptotically to the temperature corresponding to the gyroradius resolution $r_{\mathrm{L}} / \Delta x=0.6-0.8$. However, this asymptotic value is not well settled. For example, in simulations for case D, we observe fluctuations in temperature even for the marginally resolved $r_{\mathrm{L}}$, when $r_{\mathrm{L}} / \Delta x \approx 1.05$. Since we use Maxwellian plasma and calculate $r_{\mathrm{L}}$ from the perpendicular thermal velocity, we 

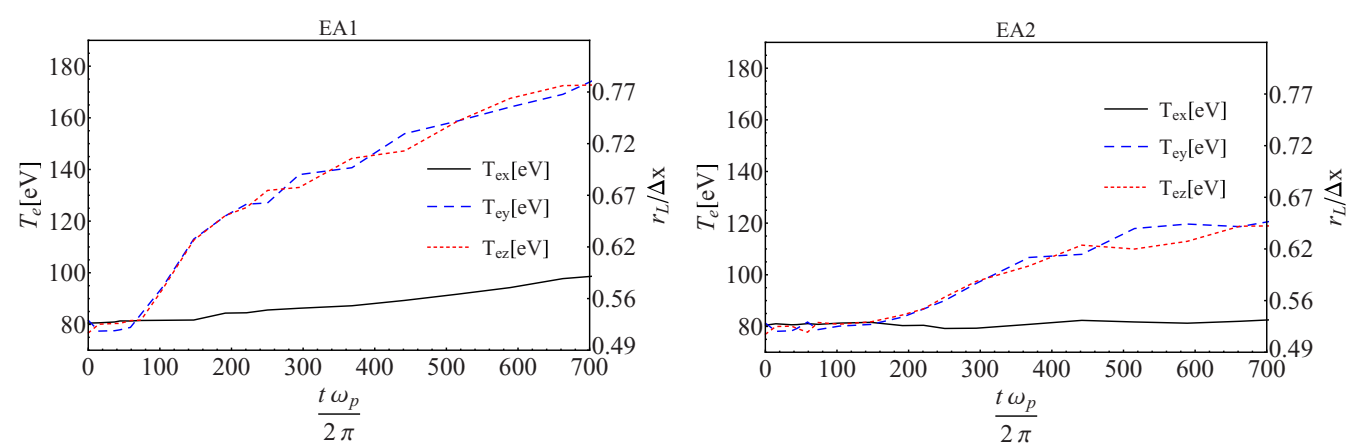

FIG. 5. The electron temperature evolution for the addtional simulations with drifting plasma. The left panel shows temperature evolution for the first-order weighting while the right panel shows temperature for the second-order weighting. The magnetic field is oriented in the $\hat{\mathbf{x}}$ direction, and the electric field in $\hat{\mathbf{y}}$ direction. It is clear that in these simulations, the second-order weighting only reduces the growth of temperature.

still have a part of particles with a smaller gyroradius than what would correspond to the thermal velocity. The exact value of this threshold likely depends on other simulation parameters and can be a subject of further research.

The need for resolving the electron gyroradius, and the problem of artificial heating of electrons for unresolved $r_{\mathrm{L}}$, were not addressed before, and only a few comments on this issue can be found in the literature, [17,19]. Melzani et al. [19] briefly mentioned electron heating during simulations with unresolved electron gyroradius using electromagnetic PIC code. They also suggested that it may be because of aliases on the grid and noted that such a parametric range should be avoided, however, no analysis of the problem was provided there. Note that in this study, we use the electrostatic PIC code, which confirms that the problem is of a general character. We also demonstrate that aliasing with the grid does not lead to unstable solutions in the linear analysis.

In a more general context of the Debye length resolution, Cormier-Michel et al. [13] discussed the artificial heating related to the order of weighting function. Parker and Birdsall [16] studied the resolution of gyromotion from the perspective of temporal resolution of the gyrofrequency, and observed that nonresolving of gyrofrequency does not affect simulation stability and causes only errors in particle orbits. On the other hand, in such systems where the electron Debye length is smaller than the electron gyroradius, the need of gyroradius resolution is automatically fulfilled due to numerical stability requirement of resolving the Debye length. This criterion is fulfilled in most weakly magnetized plasmas.

Our results show that the important aspect is the weighting function used in simulations. In cases where we observe heating due to the unresolved gyroradius, we observed it only for cases with the first (linear) order weighting function. There was no heating nor growth in potential fluctuations for the simulations with the second-order weighting. However, we verified this effect also for simulations of plasma in $\mathbf{E} \times \mathbf{B}$ fields, observing that the artificial heating as well as the growth in potential fluctuations were not fully eliminated but only lowered. Thus it is clear that the order of weighting function is important in diminishing the effect, but it is not the solution to the problem. The importance of the choice of weighting function was also discussed in Ref. [13] in the context of the Debye length resolution, where the same conclusion was reached: the higher-order weighting causes lower heating but can not fully eliminate it.

The artificial electron heating can be related to the growth in potential fluctuations and creating filament structures in the potential distribution at very small scales of the order of the grid spacing. These filaments are only present in simulations with unresolved gyroradius. The origin of the filaments is unclear, but it is likely that they are due to initial fluctuations and the fact that for unresolved gyroradius, some gyrating electrons will always be at a certain distance from the grid point. In case $\mathrm{A}$, where $\Delta x / r_{\mathrm{L}} \approx 5$, only a small fraction of electrons will have a gyromotion that will allow them to change a grid cell. Consequently, the electrons will move only along the magnetic field lines, but in a periodic system this will eventually lead to clumping and regions of enhanced potential that will scatter particles across magnetic field lines. The resulting filaments are indications of strong gradients in density which also lead to strong gradients in perpendicular components of the electric field.

Strong electric field gradients can lead to instablity and subsequently to electron heating [26]. We checked our gradients in perpendicular components of the electric field and found that instability threshold $\nabla E q / m \Omega^{2} \geqslant 1$ is not reached in our simulation and we have $\nabla E q / m \Omega^{2} \approx 0.007$. We also do not observe transition from the Maxwellian distribution to ring distribution as it was described by the authors of Ref. [26].

With steep gradients in density, one can expect that the drift waves would be present. The classical analysis of the kinetic drift waves in a collisionless system leads to the following criterion for the wave to be unstable [27,28]:

$$
k_{\perp}^{2} r_{\mathrm{Ls}}^{2}>\left(k_{\|} / k_{\perp}\right)^{2} C_{\mathrm{s}}^{2} / v_{\mathrm{de}}^{2},
$$

where $C_{s}=\sqrt{k T_{\mathrm{e}} / m_{\mathrm{i}}}$ is the sound speed, $k_{\perp}$ and $k_{\|}$are the wave vectors perpendicular and parallel to the magnetic field, $r_{\mathrm{Ls}}=m_{\mathrm{i}} v_{\mathrm{th}, \mathrm{e}} / q B$ is the effective Larmor radius for ions at electron thermal temperature, and $v_{\mathrm{de}}=C_{\mathrm{s}}^{2} / \omega_{\mathrm{ci}} L$, where $L=n / \nabla n$ is the characteristic length scale. Under the usual conditions, $v_{\mathrm{de}} \ll C_{\mathrm{s}}$ this condition is fulfilled only for $k_{\|} \ll$ $k_{\perp}$, i.e., for very long perpendicular wavelengths. However, in our case the very short $L$ leads, after some calculus, to an estimate: $0.2 k_{\perp}^{4}>0.01 k_{\|}^{2}$. Hence, the instability would also 
be present for very short perpendicular wavelengths. High frequency oscillations could couple to and excite the modes near the electron gyrofrequency as observed in Fig. 4 and lead to electron heating in the perpendicular direction, while smoothing out the gradients. In fact, in the frequency spectra in Fig. 4, we observe mostly two characteristic modes. First are oscillations on ion plasma frequency. The second are waves in the range of electron gyrofrequency and/or upper hybrid frequency. The upper hybrid waves (Bernstein modes) are typical for perpendicular propagation in magnetized plasmas and also resulted from our linear theoretical analysis. Note that these strong and short gradients on the order of Debye length are unphysical and are the result of nonresolved gyroradius.

The linear dispersion analysis (in Sec. IV and in the Appendix) shows no growth of waves in the directions perpendicular to the external magnetic field. This result is independent of the order of weighting function as well as of the resolution of the gyroradius. The gridded dispersion relation was derived assuming perturbations of the first order only, thus the theoretical analysis was based on the linear approach, which is the only treatable analytical approach. Because the onset of temperature growth is after 60-80 plasma periods we suggest that the temperature and potential growth is connected with a nonlinear phenomenon, which cannot be, in principle, reflected in the linear dispersion relation.

\section{CONCLUSION}

We studied the importance of the electron gyroradius resolution on the grid in electrostatic PIC simulations in cases where the electron gyroradius is smaller than the Debye length. We found that the simulations with unresolved gyroradius, when $\Delta x>r_{\mathrm{L}}$, show heating of electrons in directions perpendicular to the magnetic field. This heating is likely caused by nonlinear wave-particle interaction where the origin of the waves is related to the characteristic scales of density irregularities. The unphysical filamentation at scales of the order of the Debye length leads to strong density gradients that could trigger electron drift waves that could be unstable even at high frequencies. We identified two characteristic frequencies that are dominant in the spectra: (i) ion plasma frequency and (ii) gyrofrequency and its harmonics. The numerical heating of electrons can be avoided by resolving the gyroradius, or under some conditions supressed by using the second-order weighting.

\section{ACKNOWLEDGMENTS}

The authors thank Prof. Hans Pécseli and Prof. Petr Kulhánek for discussions. M.H. acknowledges Grant No. GAČR 14-37086G \& Praemium Academiae award of the CAS. V.D. acknowledges CTU internal Grant No. SGS15/073/OHK3/1T/13. W.M. was supported by the Research Council of Norway Grant No. 240000. The simulations are carried out through project Notur NN9299K. This research is a part of the 4DSpace Strategic Research Initiative at the University of Oslo.

\section{APPENDIX: DERIVATION OF THE DISPERSION RELATION ON GRID}

We start with the Vlasov equation for magnetized plasmas:

$$
\frac{\partial f_{\alpha}}{\partial t}+\left(\mathbf{v}_{\alpha} \cdot \nabla_{\mathbf{x}}\right) f_{\alpha}+\left[\frac{q_{\alpha}}{m_{\alpha}}\left(\mathbf{E}+\mathbf{v}_{\alpha} \times \mathbf{B}\right) \cdot \frac{\partial}{\partial \mathbf{v}}\right] f_{\alpha}=0,
$$

where $\alpha$ is an index denoting plasma species, $\mathbf{E}=$ $\left(E_{1 \mathrm{x}}, E_{1 \mathrm{y}}, E_{1 \mathrm{z}}\right)$, and $\mathbf{B}=\left(0,0, B_{0}\right)$. Such an equation is analytically solvable using the method of unperturbed orbits described by the authors of Ref. [29]. To find dispersion relation on the grid, we use the following grid quantities (due to the gridded Fourier transform) described by the authors of Ref. [4]:

$$
\boldsymbol{\kappa}=\left[k_{\mathrm{x}} \operatorname{dif}\left(k_{\mathrm{x}} \Delta x\right), k_{\mathrm{y}} \operatorname{dif}\left(k_{\mathrm{y}} \Delta y\right), k_{\mathrm{z}} \operatorname{dif}\left(k_{z} \Delta \mathrm{z}\right)\right],
$$

and

$$
\begin{aligned}
\mathcal{K}^{2}(\mathbf{k})= & k_{\mathrm{x}}^{2} \operatorname{dif}^{2}\left(\frac{k_{\mathrm{x}} \Delta x}{2}\right)+k_{\mathrm{y}}^{2} \operatorname{dif}^{2}\left(\frac{k_{\mathrm{y}} \Delta y}{2}\right) \\
& +k_{\mathrm{z}}^{2} \operatorname{dif}^{2}\left(\frac{k_{\mathrm{z}} \Delta z}{2}\right),
\end{aligned}
$$

which turn the Poisson equation into

$$
\rho(\mathbf{k})=\mathcal{K}^{2}(\mathbf{k}) \phi(\mathbf{k}),
$$

and the equation for the electric field into

$$
\mathbf{E}(\mathbf{k})=-\mathrm{i} \kappa(\mathbf{k}) \phi(\mathbf{k}) .
$$

Using these grid quantities and combining the methods described in Refs. [29] and [4] we can express the susceptibility for the plasma component as follows:

$$
\chi_{\alpha}=\frac{\omega_{\mathrm{p} \alpha}^{2}}{\mathcal{K}^{2}\left(\mathbf{k}_{p}\right) v_{\mathrm{th} \alpha}^{2}}\left[1+\frac{\omega \exp \left(-\lambda_{\alpha}\right)}{\sqrt{2}\left|\kappa_{z}\right| v_{\mathrm{th} \alpha}} \sum_{m=-\infty}^{\infty} \mathrm{I}_{m}\left(\lambda_{\alpha}\right) \mathrm{Z}\left(\zeta_{\alpha}^{m}\right)\right]
$$

where

$$
\lambda_{\alpha}=\left(\kappa_{\mathrm{y}} r_{\mathrm{L} \alpha}\right)^{2}, \zeta_{\alpha}^{m}=\frac{\omega+m \Omega_{\alpha}}{\sqrt{2}\left|\kappa_{z}\right| v_{\mathrm{th} \alpha}}, r_{\mathrm{L} \alpha}=\frac{m_{\alpha} v_{\mathrm{th} \alpha}}{q_{\alpha} B},
$$

$\mathrm{I}_{m}$ stands for the $m$ th mode of the Bessel function $I$, $\mathrm{Z}\left(\zeta_{\alpha}^{m}\right)$ stands for the plasma dispersion function with argument $\zeta_{\alpha}^{m}$, and $\mathbf{k}_{p}=\mathbf{k}-p \mathbf{k}_{\mathrm{g}}$, where $\mathbf{k}_{\mathrm{g}}=\left(k_{\mathrm{gx}}, k_{\mathrm{gy}}, k_{\mathrm{gz}}\right)$, and $k_{\mathrm{gx}}=2 \pi / \Delta x, k_{\mathrm{gy}}=2 \pi / \Delta y$, and $k_{\mathrm{gz}}=2 \pi / \Delta z$, and $p$ is the periodicity coefficient resulting from Fourier transformation [4]. Note that in this linear analysis the temporal and spatial derivatives within the Vlasov equation are calculated exactly, while the electric field and potential are calculated using a gridded Poisson equation. This is because the particles can move freely within the simulation box, while the electric field is calculated only at gridpoints. We can substitute the susceptibility (A6) into the equation for charge density on the grid

$$
\rho(\mathbf{k})=\sum_{\alpha} q_{\alpha} \sum_{p=-\infty}^{\infty} S\left(\mathbf{k}_{p}\right) n_{\alpha}\left(\mathbf{k}_{p}\right)
$$


using the expression for particle density

$$
n_{\alpha}\left(\mathbf{k}_{p}, \omega\right)=-S\left(\mathbf{k}_{p}\right) \frac{\mathcal{K}^{2}\left(\mathbf{k}_{p}\right) \phi\left(\mathbf{k}_{p}, \omega\right)}{q_{\alpha}} \chi_{\alpha}\left(\mathbf{k}_{p}, \omega\right)
$$

to get

$$
\rho(\mathbf{k})=-\sum_{\alpha} \sum_{p=-\infty}^{\infty}\left|S\left(\mathbf{k}_{p}\right)\right|^{2} \frac{\omega_{\mathrm{p} \alpha}^{2}}{v_{\mathrm{th} \alpha}^{2}}\left[\frac{\omega \exp \left(-\lambda_{\alpha}\right)}{\sqrt{2}\left|\kappa_{\mathrm{z}}\right| v_{\mathrm{th} \alpha}} \sum_{m=-\infty}^{\infty} \mathrm{I}_{m}\left(\lambda_{\alpha}\right) \mathrm{Z}\left(\zeta_{\alpha}^{m}\right)\right] \phi(\mathbf{k}, \omega) .
$$

Finally, using the Poisson equation (A4) we get the dispersion relation

$$
1+\sum_{\alpha} \sum_{p=-\infty}^{\infty}\left|S\left(\mathbf{k}_{p}\right)\right|^{2} \frac{\omega_{\mathrm{p} \alpha}^{2}}{\mathcal{K}^{2}\left(\mathbf{k}_{p}\right) v_{\mathrm{th} \alpha}^{2}}\left[\frac{\omega \exp \left(-\lambda_{\alpha}\right)}{\sqrt{2}\left|\kappa_{\mathrm{Z}}\right| v_{\mathrm{th} \alpha}} \sum_{m=-\infty}^{\infty} \mathrm{I}_{m}\left(\lambda_{\alpha}\right) \mathrm{Z}\left(\zeta_{\alpha}^{m}\right)\right]=0,
$$

which can be written in the usual form

$$
\varepsilon=1+\sum_{\alpha} \chi_{\alpha}=0
$$

[1] S. T. Wu and M. Dryer, Science China Earth Sciences 58, 839 (2015).

[2] M. Shoucri, Communications in Nonlinear Science and Numerical Simulation 13, 174 (2008).

[3] R. W. Hockney and J. W. Eastwood, Computer Simulation Using Particles, 1st ed. (IOP, Bristol, England, 1988).

[4] C. K. Birdsall and A. B. Langdon, Plasma Physics via Computer Simulations, 1st ed. (McGraw-Hill, New York, 1985).

[5] J. P. Boris, in Proceedings of Fourth Conference on Numerical Simulation of Plasmas (Naval Res. Lab., 1970), pp. 3-67.

[6] H. Qin, S. Zhang, J. Xiao, J. Liu, Y. Sun, and W. M. Tang, Phys. Plasmas 20, 084503 (2013).

[7] F. J. R. Simoes, M. V. Alves, and R. Gaelzer, J. Geophys. Res. 115, A06105 (2010).

[8] B. Kakad, A. Kakad, and Y. Omura, J. Geophys. Res.: Space Phys. 119, 5589 (2014).

[9] I. Sohn, J. Kim, J. Bae, and J. Lee, IEEE Transactions on Plasma Physics 44, 1823 (2016).

[10] A. A. Ganjovi, Journal of Theoretical and Applied Physics 10, 61 (2016).

[11] J. P. Verboncoeur, Plasma Phys. Controlled Fusion 47, A231 (2005).

[12] V. K. Decyk, Comput. Phys. Commun. 87, 87 (1995).

[13] E. Cormier-Michel, B. A. Shadwick, C. G. R. Geddes, E. Esarey, C. B. Schroeder, and W. P. Leemans, Phys. Rev. E 78, 016404 (2008).

[14] R. F. Abdul and R. L. Mace, Phys. Plasmas 22, 102107 (2015).
[15] G. Lapenta, J. Comput. Phys. 231, 795 (2012).

[16] S. E. Parker and C. K. Birdsall, J. Comput. Phys. 97, 91 (1991).

[17] M. Horký and W. J. Miloch, Phys. Plasmas 22, 022109 (2015).

[18] W. J. Miloch, H. L. Pécseli, and J. K. Trulsen, Plasma Phys. Controlled Fusion 55, 124006 (2013).

[19] M. Melzani, C. Winisdoerffer, R. Walder, D. Folini, J. M. Favre, S. Krastanov, and P. Messmer, Astron. Astrophys. 558, A133 (2013).

[20] W. J. Miloch, M. Kroll, and D. Block, Phys. Plasmas 17, 103703 (2010).

[21] W. J. Miloch, S. R. Habbal, and R. Esser, Astrophys. J. 752, 85 (2012).

[22] D. Block and W. J. Miloch, Plasma Phys. Controlled Fusion 57, 014019 (2015)

[23] V. Vahedi and M. Surendra, Comput. Phys. Commun. 87, 179 (1995).

[24] H. R. Skullerud, J. Phys. D: Appl. Phys. 1, 1567 (1968).

[25] M. Horký and W. J. Miloch, J. Phys.: Conf. Series 591, 012034 (2015).

[26] M. Balikhin, M. Gedalin, and A. Petrukovich, Phys. Rev. Lett. 70, 1259 (1993).

[27] H. L. Pécseli, Waves and Oscillations in Plasmas, 1st ed. (CRC, London, 2013).

[28] R. J. Goldston and P. H. Rutherford, Introduction to Plasma physics, 1st ed. (IOP, Bristol, England, 1995).

[29] S. P. Gary, Theory of Space Plasma Microinstabilities, 1st ed. (Cambridge University Press, Cambridge, England, 1993). 